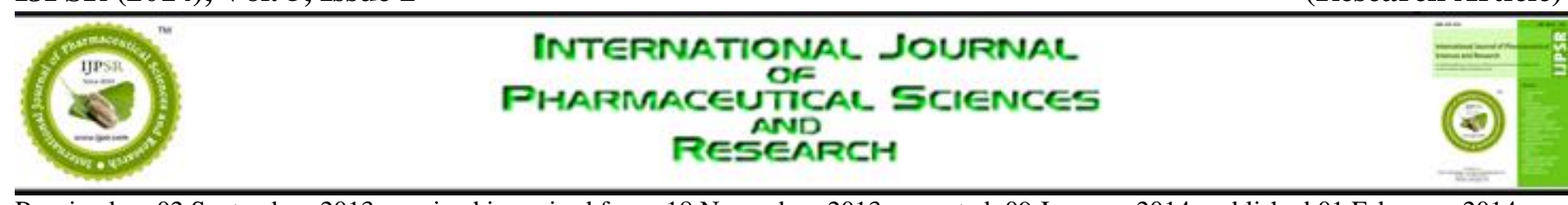

Received on 02 September, 2013; received in revised form, 18 November, 2013; accepted, 09 January, 2014; published 01 February, 2014

\title{
FORMULATION AND IN-VITRO EVALUATION OF GEL CONTAINING ETHOSOMES ENTRAPPED WITH ETODOLAC
}

Pavan Kumar Chintala* and J. Padmapreetha

Department of Pharmaceutics, KMCH College of Pharmacy, Coimbatore, Tamil Nadu, India

Keywords:

Etodolac, Soya Phosphotidyl choline, Ethanol

Correspondence to Author:

Pavan Kumar Chintala

Department of Pharmaceutics, KMCH College of Pharmacy, Coimbatore, Tamil Nadu, India

E-mail:

pavanchowdary.chintala@gmail.com

\begin{abstract}
Etodolac is an indole acetic acid derivative having halflife of 4 to $7 \mathrm{~h}$ and used for the treatment Rheumatoid arthritis. The oral use of Etodolac is not much recommended as it has many systemic side effects. The entrapment of drug in a vesicle has shown improved delivery of drug at the targeted site and has also reduced the dose and thus, has shown better patient compliance. Ethosomes are lipid vesicular carriers containing ethanol which provides better penetration of drug into the skin. Ethosomes of Etodolac were prepared by hot method. The composition includes phospholipid, ethanol, propylene glycol and distilled water. Liposomes of Etodolac were also prepared by thin film hydration technique. Selected formulations were subjected to sonication for reducing the vesicle size. FT-IR study confirmed the purity of drug and revealed no interaction between the drug and excipients. Ethosomes and liposomes were characterized for vesicle shape, vesicle size, entrapment efficiency percentage, in vitro drug diffusion. \%CDR after $8 \mathrm{~h}$ for ethosomal, liposomal are $76.55 \pm 0.70 \%, 65.61 \pm 0.68 \%$ and respectively. Ethosomal formulation (F8) was found stable at $4 \pm 2{ }^{\circ} \mathrm{C}$ and at room temperature during the storage of 45 days. Efficient delivery of drug to deep skin strata from ethosomal drug application found to be highly beneficial in localizing the drug to desired site in the skin and reduced the side effects associated with conventional treatments.
\end{abstract}

INTRODUCTION: The skin covers a total surface area of approximately $1.8 \mathrm{~m}^{2}$ and provides the contact between the human body and the external environment.

Dermal drug delivery is the topical application of drugs to the skin in the treatment of skin diseases and other inflammatory conditions.

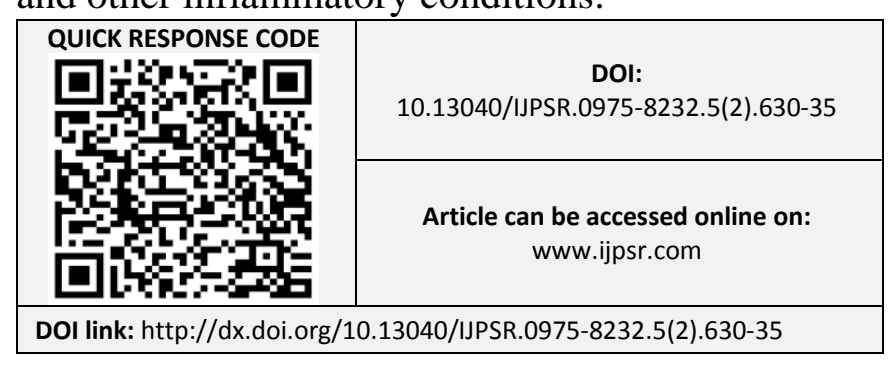

This has the advantage that high concentrations of drugs can be localized at the site of action, reducing the systemic side effects. Transdermal drug delivery uses the skin as an alternative route for the delivery of systemically acting drugs. The structure of stratum corneum is often compared with a brick wall, with the corneocytes as the bricks surrounded by the mortar of the intercellular lipid lamellae.

Many techniques have been aimed to disrupt and weaken the highly organized intercellular lipids in an attempt to enhance drug transport across the intact skin. One of the most controversial methods is the use of vehicle formulations as skin delivery systems ${ }^{1}$. 
Ethosomes are novel carrier system used for delivery of drugs having low penetration through the biological membrane mainly skin. Ethosomes are the slight modification of well-established drug carrier liposome. Ethosomes are lipid vesicles containing phospholipids, alcohol (ethanol and isopropyl alcohol) in relatively high concentration and water. ${ }^{2}$ Ethosomes are soft vesicles made of phospholipids and ethanol (in higher quantity) and water. The size range of ethosomes may vary from tens of nanometers to microns $(\mu)^{3}$.

Etodolac belongs to a class of drugs called nonsteroidal anti-inflammatory drugs (NSAIDs) ${ }^{4}$. These drugs are used for the management of mild to moderate pain, fever, and inflammation. They work by reducing the levels of prostaglandins, which are chemicals that are responsible for pain and the fever and tenderness that occur with inflammation ${ }^{5}$. Etodolac blocks the enzyme that makes prostaglandins (cyclooxygenase), resulting in lower concentrations of prostaglandins. As a consequence, inflammation, pain and fever are reduced.

\section{MATERIALS AND METHODS:}

Materials: Etodolac was obtained as a gift sample from Rachem Pharmaceuticals Ltd., Hyderabad. Soya Phosphotidyl choline From Himedia mumbai. Ethanol and Propylene glycol from SD Fine chemicals Mumbai. All other chemicals and reagent were of analytical grade.

\section{Methods:}

\section{Preparation of Ethosomes:}

Hot Method: Ethosomes were prepared by hot method. In this specified amount of phospholipid was dissolved in water and kept under magnetic stirrer for $30 \mathrm{~min}$. The lipid mixture was heated at $40^{\circ} \mathrm{c}$ on magnetic stirrer latter specified amount of Etodolac was added to ethanol to this propylene glycol was added and it was kept under magnetic stirrer latter lipid solution was added drop by drop and it was kept under magnetic stirrer for $1 \mathrm{hr}$. The solution was kept at $40^{\circ} \mathrm{C}$ for $1 \mathrm{hr}$ later it was kept for sonication using ultra sonicator for $15 \mathrm{~min}$ to reduce the particle size

\section{Preparation of Liposomes ${ }^{6}$ :}

1. Lipid Film Hydration Method: Phospholipid and cholesterol were dissolved in little quantity of chloroform in a round bottom flask. The solvent was allowed to evaporate using vacuum evaporator to form a thin film of lipid on the wall of flask. Lipid film was then hydrated with the solution of drug in a phosphate buffer of $\mathrm{pH} 6.8$ by magnetic stirrer for $1 \mathrm{~h}$ at R.T.

Preparation of Gel Base ${ }^{7}: 1 \%$ w/w gel base was prepared by dispersing Carbopol 934 in distilled water containing $0.2 \%$ methyl paraben and $0.02 \%$ propyl paraben, using magnetic stirrer. Here, Carbopol 934 was used as gelling agent and methyl paraben and propyl paraben were used as preservatives.

Preparation of Ethosomal Gel ${ }^{\mathbf{8}}$ : The Ethosomal sediments were collected and incorporated into the prepared gel base to get ethosomal gel.

\section{Evaluation of Ethosomes of Etodolac:}

1. Entrapment efficiency ${ }^{\mathbf{9}}$ : $10 \mathrm{ml}$ of ethosomal solution was taken in Centrifuge tube. Ethosomal formulation was subjected to $5000 \mathrm{rpm}$ for $1 \mathrm{hr}$. Using laboratory centrifuge the unentrapped drug concentration was determined spectrophotometrically at $274 \mathrm{~nm}$. The drug entrapment percentage was calculated using the given equation.

Entrapment efficiency $\%=\mathrm{A}_{2}-\mathrm{A}_{1} / \mathrm{A}_{2} \times 100$

Where, $\mathrm{A}_{1}=$ Amount of Etodolac in sediment, $\mathrm{A}_{2}=$ Total amount of Etodolac added.

2. Vesicle size: The vesicle size of the best formulation was determined by using Dynamic light scattering.

3. Vesicle shape: The vesicle shape of best formulation was determined by using scanning electron microscopy.

4. Optical microscopy observation ${ }^{10}$ : The ethosomal dispersion was spread on the glass slide using a glass rod. Formation of 
multilamellar vesicles was confirmed by examining the ethosomal suspension under an optical microscope with the magnification power of $100 \mathrm{x}$.

5. pH studies: The $\mathrm{pH}$ values of $1 \%$ aqueous solutions of the prepared gels were measured by a $\mathrm{pH}$ meter.

6. Extrudability study ${ }^{14}$ : In the present study, the method adopted for evaluating gel formulation for extrudability was based upon the quantity in percentage of gel and gel extruded from lacquered aluminum collapsible tube on application of weight in grams required to extrude at least $0.5 \mathrm{~cm}$ ribbon of gel in 10 seconds. The extrudability was than calculated by using the following formula:

Extrudability $=$ Applied weight to extrude gel from tube (in gm) / Area (in cm2)

7. Spreadability Coefficient studies ${ }^{13}$ : The Spreadability of the gel formulations was determined $48 \mathrm{~h}$ after preparation, by measuring the spreading diameter of $10 \mathrm{~g}$ of the gel between two glass plates after 1min. The mass of the upper plate was standardized at $20 \mathrm{~g}$, placing slides one above the other and count time taken for $2^{\text {nd }}$ slide to slip out from other slide

Spreadability coefficient $=\mathrm{ML} / \mathrm{T}$

$\mathrm{M}=$ mass, $\mathrm{L}=$ length, $\mathrm{T}=$ time

8. In vitro diffusion study ${ }^{11}$ : In vitro diffusion study was carried out in a Franz diffusion cell using cellophane membrane.
The cellophane membrane was mounted on the Franz diffusion cell. Formulation was applied through donor compartment on the dialysis membrane. Reservoir compartment was filled with $25 \mathrm{ml}$ phosphate buffer of $\mathrm{pH} 6.8$ The study was carried out at $37 \pm$ $1{ }^{\circ} \mathrm{C}$ and at a speed of $100 \mathrm{rpm}$ for $8 \mathrm{~h}$. Samples were withdrawn from reservoir compartment at $1 \mathrm{~h}$ interval and absorbance was measured spectrophotometrically at $274 \mathrm{~nm}$. Each time the reservoir compartment was replenished with the same quantity of $6.8 \mathrm{pH}$ phosphate buffer.

9. Stability studies ${ }^{12}$ : Stability testing of drug products begins as a part of drug discovery and ends with the commercial product. To assess the drug and formulation stability, stability studies were done. The stability studies were carried out for the most satisfactory formulation. The most satisfactory formulation was sealed in a container and kept at $4 \pm 2^{\circ} \mathrm{C}$ and at R.T. for 45 days. At the end 45 days, the sample was analyzed for the entrapment efficiency percentage, and in vitro skin diffusion study.

RESULTS AND DISCUSSION: The present study was carried out to develop the Ethosomes of Etodolac by hot method. Hence, it was necessary to find suitable excipients with good compatibility. Ten formulations of Etodolac were prepared with different concentrations of soya phosphatidyl choline and Ethanol. Phospholipid was used as vesicle forming agent. Ethanol is used as penetration enhancer. Propylene glycol is used as viscosity forming agent. Distilled water is used as vehicle (table 1).

TABLE 1: FORMULATION CHART OF ETHOSOMES \&LIPOSOMES CONTAINING ETODOLAC

\begin{tabular}{ccccccccccc}
\hline Ingredients & F1 & F2 & F3 & F4 & F5 & F6 & F7 & F8 & F9 & F10*L \\
\hline Etodolac (mg) & 300 & 300 & 300 & 300 & 300 & 300 & 300 & 300 & 300 & 300 \\
Phospholipid (mg) & 300 & 300 & 300 & 600 & 600 & 600 & 900 & 900 & 900 & 900 \\
Ethanol (ml) & 6 & 9 & 12 & 6 & 9 & 12 & 6 & 9 & 12 & - \\
Propylene glycol (ml) & 3 & 3 & 3 & 3 & 3 & 3 & 3 & 3 & 3 \\
Cholesterol (mg) & - & - & - & - & - & - & - & - & - & 300 \\
Chloroform (ml) & - & - & - & - & - & - & - & - & - & 25 \\
Dist. Water & Upto & Upto & Upto & Upto & Upto & Upto & Upto & Upto & Upto3 & $25 \mathrm{ml}$ \\
\hline
\end{tabular}

*L=Liposomes 


\section{Pre Formulation Study:}

1. Melting Point: Melting point of Etodolac was found to be $146^{\circ} \mathrm{C}$ and which was found to be in the range of $144-150^{\circ} \mathrm{C}$.

2. Solubility: Etodolac is freely soluble in Ethanol. Sparingly soluble in $6.8 \mathrm{pH}$ phosphate buffer and insoluble in water

\section{Drug Polymer Compatibility:}

1. FTIR studies: The drug excipient compatibility study was done by using SHIMADZU FT/IR spectrometer. The IR spectra for pure drug, and drug-excipient mixture were shown in figures 7-9. The spectrum of the pure drug and excipients suggests that there were no interaction between drug and excipients and were compatible with each other.

2. Vesicle size: The vesicle size was determined by Dynamic light scattering .It was found to be $242.9 \mathrm{~nm}$ it was shown in figure 11.

3. Vesicle shape: Vesicle shape was determined by Scanning Electron Microscopy. It was shown in figure 12.
4. Entrapment efficiency: The entrapment efficiency was found to be higher in F8 formulation with $80.5 \%$. F10 formulation of liposomes showed entrapment efficiency of $69.33 \%$ which is lesser than liposomes. F8 formulation is selected as best formulation for further studies. it was shown in figure 13. The values are shown in table 2.

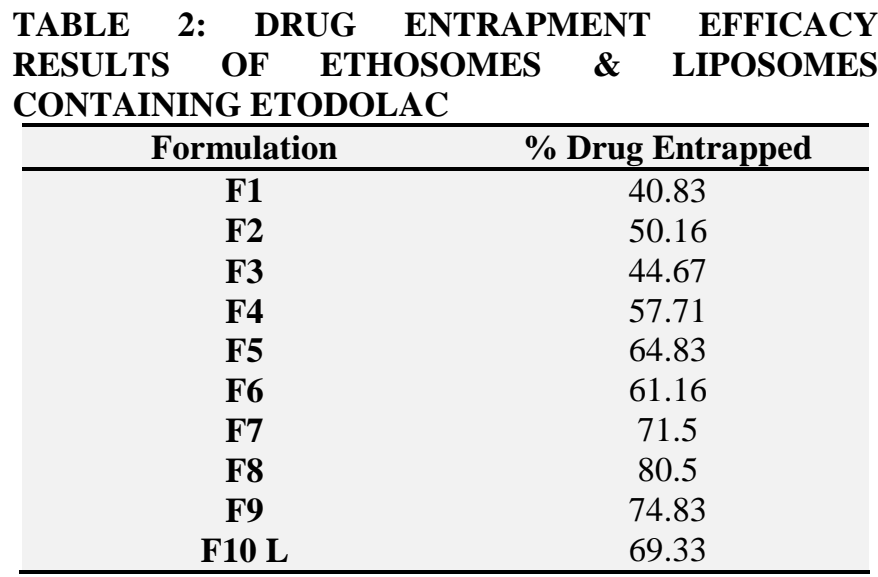

5. In-vitro Drug diffusion studies: In-vitro drug release was observed more in F8 formulation with $76.55 \%$ and drug release in liposomes was found to be $65.61 \%$ decrease in drug release when compared to ethosomes was due to absence of Ethanol. The values are shown in table 3 .

TABLE 3: IN-VITRO DRUG RELEASE

\begin{tabular}{|c|c|c|c|c|c|c|c|c|c|c|}
\hline $\begin{array}{c}\text { Time } \\
\text { (hr) }\end{array}$ & F1 & F2 & F3 & F4 & F5 & F6 & F7 & F8 & F9 & F10 $* \mathbf{L}$ \\
\hline 1 & $3.45 \pm 0.12$ & $5.43 \pm 0.21$ & $4.52 \pm 0.24$ & $14.54 \pm 0.15$ & $20.45 \pm 0.16$ & $16.85 \pm 0.2$ & $24.51 \pm 0.09$ & $32.51 \pm 0.21$ & $28.97 \pm 0.41$ & $25.02 \pm 0.17$ \\
\hline 2 & $7.62 \pm 0.23$ & $11.18 \pm 0.22$ & $7.16 \pm 0.29$ & $20.68 \pm 0.44$ & $26.08 \pm 0.19$ & $19.72 \pm 0.21$ & $28.10 \pm 0.51$ & $39.69 \pm 0.25$ & $37.04 \pm 0.59$ & $27.21 \pm 0.30$ \\
\hline 3 & $10.63 \pm 0.25$ & $16.42 \pm 0.67$ & $12.17 \pm 0.37$ & $28.57 \pm 0.13$ & $29.88 \pm 0.25$ & $27.76 \pm 0.24$ & $33.90 \pm 0.56$ & $44.71 \pm 0.27$ & $41.94 \pm 0.66$ & $34.85 \pm 0.33$ \\
\hline 4 & $16.82 \pm 0.13$ & $24.53 \pm 0.25$ & $18.59 \pm 0.25$ & $33.92 \pm 0.45$ & $37.51 \pm 0.55$ & $33.10 \pm 0.22$ & $39.93 \pm 0.55$ & $52.17 \pm 0.31$ & $49.36 \pm 0.70$ & $40.95 \pm 0.13$ \\
\hline 5 & $20.37 \pm 0.15$ & $33.75 \pm 0.29$ & $23.89 \pm 0.41$ & $38.88 \pm 0.23$ & $42.13 \pm 0.29$ & $40.94 \pm 0.2$ & $45.43 \pm 0.57$ & $56.90 \pm 0.35$ & $54.07 \pm 0.82$ & $46.75 \pm 0.17$ \\
\hline 6 & $28.718 \pm 1.52$ & $38.64 \pm 0.32$ & $29.86 \pm 0.33$ & $43.91 \pm 0.22$ & $48.52 \pm 0.28$ & $46.23 \pm 0.26$ & $49.44 \pm 0.52$ & $64.10 \pm 0.12$ & $61.53 \pm 0.95$ & $50.92 \pm 0.18$ \\
\hline 7 & $34.35 \pm 1.42$ & $43.16 \pm 0.29$ & $36.53 \pm 1.08$ & $48.31 \pm 0.25$ & $55.03 \pm 0.22$ & $51.33 \pm 0.25$ & $57.75 \pm 0.09$ & $71.17 \pm 0.16$ & $66.80 \pm 1.46$ & $59.17 \pm 0.29$ \\
\hline 8 & $37.59 \pm 0.68$ & $47.73 \pm 0.77$ & $41.49 \pm 0.89$ & $53.40 \pm 0.94$ & $61.45 \pm 0.95$ & $57.46 \pm 0.92$ & $67.44 \pm 0.88$ & $76.55 \pm 0.70$ & $72.50 \pm 0.72$ & $65.61 \pm 0.68$ \\
\hline
\end{tabular}

6. FTIR of Ethosomes: The FTIR report of best formulation of Ethosomes showed same characteristic bands as pure drug this confirm that drug Etodolac was incorporated in ethosomes. It is shown in figure 10.

7. pH studies: The $\mathrm{pH}$ of the best formulation was found to be 5.5 which was found to be in the acidic range. The gels that are applied to skin should be acidic in nature because the skin $\mathrm{pH}$ is acidic in nature.

8. Spreadability coefficient studies: The Spreadability coefficient of the best formulation was found to be $7.2 \mathrm{gm} \mathrm{cm} / \mathrm{sec}$. 
9. Extrudability studies: The Extrudability of the best formulation was found to be $1.12 \mathrm{gm} / \mathrm{cm}^{2}$.

10. Release kinetics: The release kinetics of ethosomes of Etodolac showed that the drug follows higuchi release and it follows non fickian release. There is no significance difference observed in other values. The values are shown in table 4 . It was shown in figures $4 \& 5$.

TABLE 4: KINETIC MODELING DATA

\begin{tabular}{cccccc}
\hline \multirow{2}{*}{ Formulation } & Zero order & First order & Higuchi model & \multicolumn{2}{c}{ Korsemeyer-Peppas } \\
\cline { 2 - 6 } & $\mathbf{r}^{\mathbf{2}}$ & $\mathbf{r}^{\mathbf{2}}$ & $\mathbf{r}^{\mathbf{2}}$ & slope & $\mathbf{r}^{\mathbf{2}}$ \\
\hline F1 & 0.957 & 0.978 & 0.994 & 0.533 & 0.965 \\
F2 & 0.966 & 0.986 & 0.992 & 0.633 & 0.996 \\
F3 & 0.990 & 0.899 & 0.978 & 1.255 & 0.984 \\
F4 & 0.992 & 0.859 & 0.954 & 1.539 & 0.978 \\
F5 & 0.986 & 0.861 & 0.923 & 1.170 & 0.993 \\
F6 & 0.899 & 0.993 & 0.975 & 0.442 & 0.982 \\
F7 & 0.909 & 0.989 & 0.996 & 0.415 & 0.970 \\
F8 & 0.939 & 0.970 & 0.987 & 0.481 & 0.938 \\
F9 & 0.981 & 0.969 & 0.924 & 0.673 & 0.986 \\
\hline
\end{tabular}

11. Stability studies: There was no significant change observed in entrapment efficiency and in-vitro drug release studies for the best formulation after 45 days the values are shown in table $5 \&$ 6. It was shown in figures 6 \& 17.

TABLE 5: ENTRAPMENT EFFICIENCY \% AFTER STABILITY STUDIES

\begin{tabular}{ccc}
\hline $\begin{array}{c}\text { Formulation } \\
\text { code }\end{array}$ & \multicolumn{2}{c}{$\begin{array}{c}\text { Entrapment efficiency \% after } \\
\text { stability studies }\end{array}$} \\
\hline \multirow{2}{*}{ F8 } & At $\mathbf{4} \pm \mathbf{2}^{\circ} \mathbf{C}$ & At R.T. \\
& 79.16 & 76.33 \\
\hline
\end{tabular}

TABLE 6: COMPARATIVE DIFFUSION PROFILE AFTER STABILITY STUDIES

\begin{tabular}{ccc}
\hline \multirow{2}{*}{ TIME (h) } & \multicolumn{2}{c}{ \% CDR } \\
\cline { 2 - 3 } & $\mathbf{A t ~} \mathbf{4} \pm \mathbf{2}^{\circ} \mathbf{C}$ & At R.T. \\
\cline { 2 - 3 } $\mathbf{~}$ & $\mathbf{F 8}$ & $\mathbf{F 8}$ \\
\hline $\mathbf{2}$ & $22.15 \pm 0.63$ & $20.15 \pm 0.63$ \\
$\mathbf{3}$ & $28.75 \pm 0.57$ & $26.94 \pm 0.74$ \\
$\mathbf{4}$ & $38.81 \pm 0.67$ & $35.81 \pm 0.63$ \\
$\mathbf{5}$ & $46.45 \pm 0.62$ & $43.71 \pm 0.68$ \\
$\mathbf{6}$ & $52.62 \pm 0.47$ & $49.41 \pm 0.58$ \\
$\mathbf{7}$ & $61.13 \pm 0.51$ & $57.81 \pm 0.68$ \\
$\mathbf{8}$ & $65.72 \pm 0.61$ & $63.72 \pm 0.69$ \\
\hline
\end{tabular}

SUMMARY: A novel ethosomal system has been developed for transdermal delivery. Etodolac is generally given by oral route however it has several adverse effects like gastric ulceration bleeding and first pass metabolism etc. To overcome these limitations, alternative transdermal route has been selected. Its permeability is increased by using ethanol in the formulation in the present work ethosomes of Etodolac were prepared by hot method and evaluated. Liposomes were prepared by thin film lipid hydration method. The prepared ethosomes were characterised for entrapment efficiency, vesicle size, vesicle shape, compatibility studies and stability studies. Ethosomal gel was evaluated for in vitro drug release, $\mathrm{pH}$, and Spreadability studies. Thus, the prepared ethosomes was proved to be potential candidate for Transdermal delivery system.

CONCLUSION: From the experimental results it can be conclude that. From the IR spectra it can be revealed that there was no interaction between drug and excipients hence they are compatible. \% Entrapment efficiency and in vitro drug release was higher for F8 formulation. Particle size was analysed by DLS and found that to be $242 \mathrm{~nm}$ Vesicle shape was analysed by SEM. From all the parameters it can be conclude that ethosomes are better than liposomes.

As the concentration of ethanol increases \% drug release and \%entrapment efficiency decreased. As the concentration of Phospholipid increases \%drug release and \%entrapment efficiency increased. Optimized formulation followed Higuchi plot and non-Fickian release. It can be conclude that ethosomes are potential candidate for Transdermal drug delivery. 


\section{REFERENCES:}

1. Krijavainen M, Urtti A Jaaskelainen I, Suhonen T.M, Paronen P, Valjakka Koskela R, Monnokonen J. Interaction of liposomes with human skin in vitro- the influence of lipid composition and structure. 1996; 1304: 179-189

2. Patel S: Ethosomes: A promising tool for transdermal delivery of drug, Pharma Info.Net. 2007; 5(3).

3. Touitou E, Dayan N, Bergelson L, Godin B and Eliaz M: Ethosomes novel vesicular carriers for enhanced delivery: characterization and skin penetration properties, J Control Release. 2000; 65: 403-418.

4. Gaston GW, Mallow RD, Frank JE. The efficacy of Etodolac for patients with pain following oral surgery. $J$ Oral Maxillofac Surg. 1984 Jun; 42(6):362-366.

5. Hutton CE. The effectiveness of 100 and $200 \mathrm{mg}$ Etodolac (Ultradol), aspirin, and placebo in patients with pain following oral surgery. Oral Surg Oral Med Oral Pathol. 1983 Dec; 56(6):575- 580.

6. BarupalAk, Ramteke S. Preparation and characterization of Ethosomes for topical delivery of Aceclofenac. IntJPharm Sci. 2010; 72(5):582 -6.
7. Senthilkumar KL, Ezhilmuthu RP, Praveen P. Preparation and characterization of nabumetone liposomes. Int $\mathbf{J}$ Life sci Bio PharmaRes.2012; 1 (1):81-86.

8. M.K Bhalaria, Naik S, A.N Misra, Ethosomes A novel Delivery system for anti-fungal drugs in the treatment of topical fungal disease. IndJ Exp Bio. 2009; (47):368-75.

9. Maurya SD. Enhanced Transdermal Permeation of indinavirsulphate through stratum corneumvia, Novel permeation enhancers: Ethosomes. Der pharmaciaLettre. 2010; 2(5):208-220.

10. Vivek Dave, Dhirendra Kumar, Shaila Lewis, Sarvesh Paliwal. Ethosome for Enhanced Transdermal Drug Delivery of Aceclofenac. International Journal of Drug Delivery 2 (2010), 81-92.

11. Mamta B, Anar J, shah R.An overview of ethosomes as advanced herbal drug delivery system.IJRRPAS.2(1): 1-14.

12. Pratima NA, Shailee T, Ethosomes: A Novel Tool for Transdermal Drug Delivery. Int J Res Phar Sci. 2012; 2(1):1-20.

13. Rathore RPS, Nema RK, Formulation and evaluation of topical gels of Ketoprofen Asian. J. Pharm. Clinical. Res., 1: $12-16,(2008)$.

\section{How to cite this article:}

Chintala PK and Padmapreetha J: Formulation and in-vitro evaluation of gel containing ethosomes entrapped with etodolac. Int J Pharm Sci Res 2014; 5(2): 630-35.doi: 10.13040/IJPSR. 0975-8232.5(2).630-35

All $\odot 2013$ are reserved by International Journal of Pharmaceutical Sciences and Research. This Journal licensed under a Creative Commons Attribution-NonCommercial-ShareAlike 3.0 Unported License.

This article can be downloaded to ANDROID OS based mobile. Scan QR Code using Code/Bar Scanner from your mobile. (Scanners are available on Google Playstore) 